Diagnostic Microbiology and Infectious Disease 40 (2001) 121-123

Case reports
DIAGNOSTIC

MICROBIOLOGY

AND INFECTIOUS

DISEASE

www.elsevier.com/locate/diagmicrobio

\title{
Candida albicans spinal epidural abscess secondary to prosthetic valve endocarditis
}

\author{
Ja-Der Liang, Chi-Tai Fang*, Yee-Chun Chen, Shan-Chwen Chang, Kwen-Tay Luh \\ Department of Internal Medicine and Laboratory Medicine, National Taiwan University Hospital, 7 Chung-Shan South Road, Taipei 100, Taiwan
}

Received 02 January 2001; accepted 23 April 2001;

\begin{abstract}
A 56-year-old woman, with underlying rheumatic heart disease status post mitral valve replacement, presented with fever, low back pain radiating to right leg, and congestive heart failure. Magnetic resonance imaging detected an L5-S1 spinal epidural abscess. A vegetation on prosthetic mitral valve was found by transesophageal echocardiography. Cultures of epidural aspirate, surgical specimen, and blood all grew Candida albicans. She received surgical drainage of the spinal epidural abscess and i.v. amphotericin B $1 \mathrm{mg} / \mathrm{kg} / \mathrm{day}$ for eight weeks. Clinical symptoms improved gradually and she was discharged without neurologic sequelae. She remained well and continued to lead an active life two years after discharge. () 2001 Elsevier Science Inc. All rights reserved.
\end{abstract}

\section{Introduction}

Spinal epidural abscess is a neurologic emergency that requires prompt surgical drainage and appropriate antimicrobial therapy to minimize the risk of cord compression and paraplegia (Hlavin et al., 1990; Khanna et al., 1996). The most common pathogen of spinal epidural abscess is Staphylococcus aureus (Baker et al., 1975; Hlavin et al., 1990; Khanna et al., 1996); Candida albicans is extremely rare. There is only one case report of $C$. albicans spinal epidural abscess, which was secondary to the adjacent spondylodiscitis (Derkinderen et al., 2000). C. albicans spinal epidural abscess secondary to systemic infection has not yet been reported. We now report a case of $C$. albicans spinal epidural abscess that was secondary to prosthetic valve endocarditis.

\section{Case report}

A 56-year-old woman came to the outpatient department of National Taiwan University Hospital in February 1998 with a complaint of acute-onset low back pain for one week. There is no recent trauma, acupuncture or local manipulation. Physical examination was negative. Lumbar spine ra-

\footnotetext{
* Corresponding author. Tel.: +1-886-2-2312-3456; fax.: +1-886-22314-8482

E-mail address: fangct@ha.mc.ntu.edu.tw (C.T. Fang).
}

diography showed L5-S1 isthmic spondylolisthesis with spondylolysis only. Analgesics and bed-rest were prescribed. However, bilateral lower limbs weakness and radiation pain to the right lower limb gradually developed in the following one month. Further questioning of the patient revealed a history of mitral valve replacement with metallic prosthesis due to rheumatic heart disease five years ago and anticoagulant therapy with warfarin had been given since then. A second operation for thrombus removal was performed four years ago due to left atrial thrombosis and prosthetic valve dysfunction. She was hospitalized for further evaluation.

Examination at the time of admission revealed a body temperature of $39^{\circ} \mathrm{C}$, bilateral positive straight-leg-raising test and a mild decrease of right lower extremity muscle power (grade $4 / 5$ on the manual muscle test). Other findings included a metallic mitral click with a grade $2 / 6$ pansystolic murmur over left sternal border, engorgement of the jugular veins, bilateral basal lung crackles, and hepatomegaly. The hemogram showed a leukocyte count of $11460 / \mathrm{mm}^{3}$ with 77.4\% neutrophils. Magnetic resonance imaging (MRI) showed L5-S1 spondylitis and an L5-S1 epidural abscess (Fig. 1). Empiric antibiotic therapy with i.v. oxacillin $8 \mathrm{~g} /$ day and cefotaxime $6 \mathrm{~g} / \mathrm{day}$ was given. Cultures of peripheral blood (two sets) and the computed tomography (CT)guided aspirate all yielded Candida albicans. But the urine culture was negative. Antimicrobial therapy was changed to i.v. amphotericin B $1 \mathrm{mg} / \mathrm{kg} / \mathrm{day}$ and surgical drainage of the abscess was performed successfully. Cultures of the 


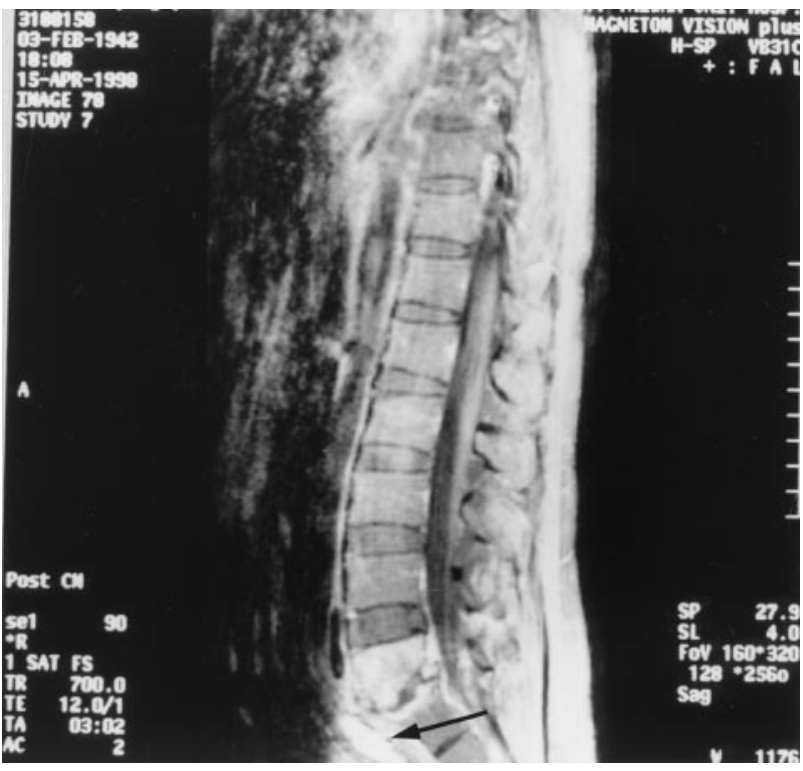

Fig. 1. Magnetic resonance image shows an epidural abscess (arrow) at the level of the L5-S1 spine.

drained pus yielded the same pathogen $C$. albicans. Molecular typing using pulsed field gel electrophoresis (PFGE) showed that all of the $C$. albicans isolates had the same PFGE pattern (Fig. 2). The radiation pain and limb weak-

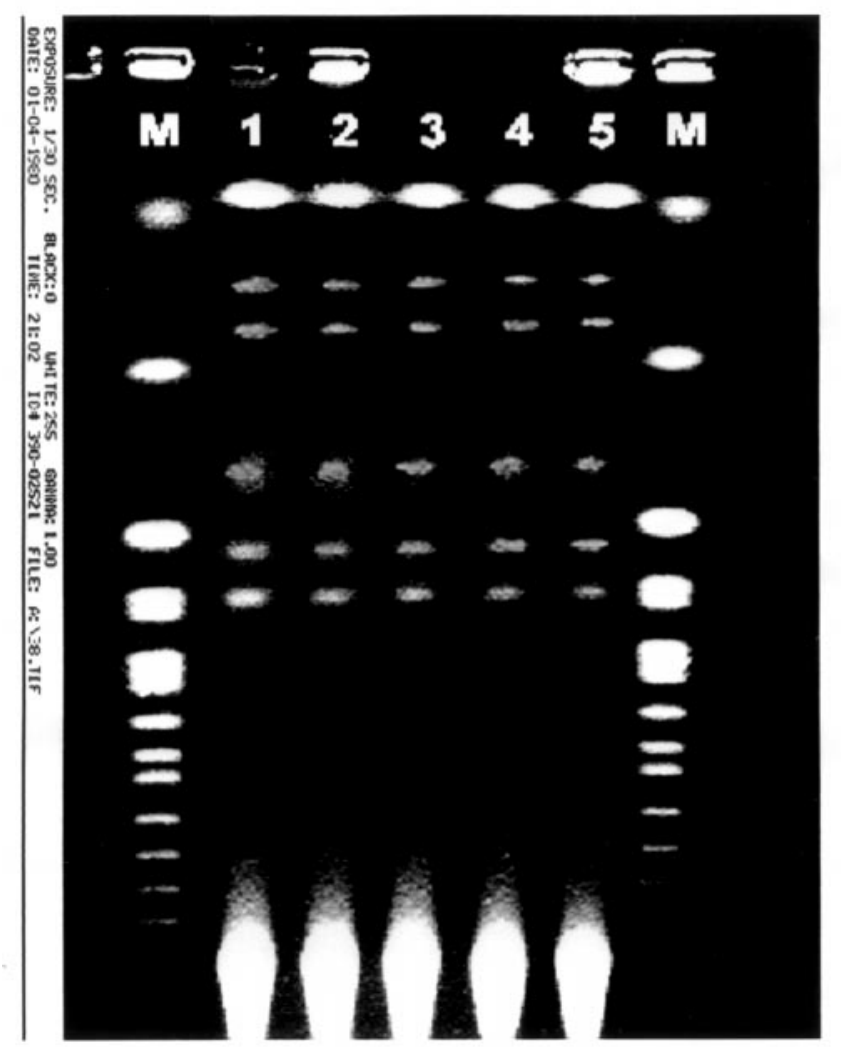

Fig. 2. Pulsed field gel electrophoresis shows all five Candida albicans isolates have the identical pattern. M: marker. ness improved after operation. However, intermittent fever persisted and congestive heart failure developed. A vegetation on the prosthetic mitral valve was detected by transesophageal echocardiography, and a Janeway's lesion on right palm with local tenderness developed during amphotericin B therapy. The minimum inhibitory concentrations of amphotericin B and fluconazole were $2 \mu \mathrm{g} / \mathrm{mL}$ and $0.5 \mu \mathrm{g} / \mathrm{mL}$ respectively, determined by the broth microdilution method (NCCLS, 1997). Amphotericin B was given for a total of eight weeks and the fever subsided gradually. Symptoms of congestive heart failure improved with diuretics. A follow-up spinal MRI showed no residual epidural abscess. She was discharged without any neurologic sequelae. Maintenance therapy with oral fluconazole $400 \mathrm{mg}$ / day was given for 12 months. Three months after discontinuation of fluconazole, a follow-up transesophageal echocardiography still showed vegetation on the prosthetic mitral valve, but simultaneous follow-up fungal blood cultures were negative. One year and 10 months after discontinuation of fluconazole, she remained well and continued to lead an active life.

\section{Discussion}

C. albicans is one of the most important fungal pathogens causing human diseases (Edward, 1999). Predisposing factors of $C$. albicans infection include major operations, indwelling catheters, prosthetic material, total parenteral nutrition, broad-spectrum antibiotics, diabetes mellitus, and immunosuppression (Edward, 1999). Our patient had received open-heart surgery twice- one for mitral valve replacement and the other for thrombus removal. She also had received central venous catheterization, parenteral nutrition and broad-spectrum antibiotics during postoperative stay at intensive care unit. These events put her at an increased risk for $C$. albicans infection.

A spinal epidural abscess may develop from local extension, hematogenous spread or from a lymphatic route (Baker et al., 1975; Hlavin et al., 1990; Khanna et al., 1996). As there was no local cutaneous lesion, previous spinal procedure, or trauma, hematogenous spread from prosthetic valve endocarditis was the most likely process in our patient. The patient had a negative urine culture before the start of antifungal therapy, which excluded the possibility of candidal colonization in the urinary tract as a focus of origin. The diagnosis of infective endocarditis in our patient was based on the Durack's criteria (Durack et al., 1994) with one major criteria (a newly documented vegetation on echocardiography) and more than three minor criteria (valvular heart disease status post prosthetic valve replacement, two sets of blood culture of C. albicans, fever more than $38^{\circ} \mathrm{C}$ and the Janeway's lesion). Her prosthetic valve endocarditis was probably related to prior open-heart surgery, despite a four-year interval, because the patient had no other risk factors. 
Although surgical intervention is usually considered necessary for fungal endocarditis (Melgar et al., 1997; Yu et al., 1994), some held opposite opinions. Lytle reviewed 1000 cardiac reoperations, and $15 \%$ of the patients died after the secondary valve reoperation (Lytle et al., 1986). Late relapses may occur despite combined prosthetic valve replacement and prolonged antifungal therapy (Johnson et al., 1991). Because of the high perioperative risk from a third open-heart surgery, amphotericin B therapy alone was given in our patient. Due to concern of recurrence, fluconazole $400 \mathrm{mg} /$ day was prescribed as maintenance suppressive therapy for 10 months. Gilbert had reviewed 34 cases of fungal prosthetic valve endocarditis successfully treated with antifungal therapy alone from 1964 to 1996 (Gilbert et al., 1996). Here we provide another successful experience.

To our knowledge, this is the first case report of $C$. albicans spinal epidural abscess secondary to $C$. albicans prosthetic valve endocarditis. With prompt surgical drainage of epidural abscess and appropriate antifungal treatment, satisfactory outcome was achieved without neurologic sequelae. C. albicans should be included in the etiologic differential diagnosis of spinal epidural abscess in patients with concurrent prosthetic valve endocarditis.

\section{References}

Baker, A. S., Ojemann, R. G., Swartz, M. N., \& Richardson, E. P. (1975). Spinal epidural abscess. New England Journal Medicine, 293, 465468.
Derkinderen, P., Bruneel, F., Bouchaud, O., \& Regnier, B. (2000). Spondylodiscitis and epidural abscess due to C. albicans. Eur Spine J, 9, $72-74$.

Durack, D. T., Lukes, A. S., \& Bright, D. K. (1994). New criteria for diagnosis of infective endocarditis: utilization of specific echocardiographic findings. American Journal of Medicine, 96, 200-209.

Edwards, J. E. (1999). Candida species, In: G. L. Mandell, J. E. Bennett \& R. Dolin (Eds.), Principles and Practice of Infectious Disease. 5th ed., (pp. 2656-2674). Churchill Livingstone, Philadelphia.

Gilbert, H. M., Peters, E. D., Lang, S. J., \& Hartman, B. J. (1996). Successful treatment of fungal prosthetic valve endocarditis: case report and review. Clin Infect Dis, 22, 348-354.

Hlavin, M. L., Kaminski, H. J., Ross, J. S., \& Ganz, E. (1990). Spinal epidural abscess: a ten-year perspective. Neurosurgery, 27, 177184.

Johnson, P. G., Domanski, M., Tucker, E., Rothenberg, M., Cunnion, R. E., Pizzo, P. A., \& Walsh, T. J. (1991). Late recurrent Candida endocarditis. Chest, 99, 1531-1533.

Khanna, R. K., Malik, G. M., Rock, J. P., \& Rosenblum, M. L. (1996). Spinal epidural abscess: evaluation of factors influencing outcome. Neurosurgery, 39, 958-964.

Lytle, B. W., Cosgrove, D. M., \& Taylor, P. C. (1986). Reoperations for valve surgery: perioperative mortality and determinants of risk for 1,000 patients, 1958-1984. Ann Thorac Surg, 42, 632-643.

Melgar, G. R., Masser, R. M., Gordon, S. M., Lytle, B. W., Keys, T. F., \& Longworth, D. L. (1997). Fungal prosthetic valve endocarditis in 16 patients: an 11-year experience in a tertiary care hospital. Medicine, 76, 94-103.

The National Committee for Clinical Laboratory Standard. (NCCLS) (1997). Reference method for broth dilution antifungal susceptibility testing for yeasts; Approved standard. NCCLS document M27-A (ISBN 1-56238-328-0). NCCLS, 940 West Valley Road, Suite 1400, Wayne, Pennsylvania 19087.

Yu, V. L., Fang, G. D., \& Keys, T. F. (1994). Prosthetic valve endocarditis: superiority of surgical valve replacement versus medical therapy only. Ann Thorac Surg, 58, 1073-1077. 